\title{
FASCEÍTE NECROSANTE CÉRVICO-FACIAL DE ORIGEM ODONTOGÊNICA DA ORIGEM AO TRATAMENTO: UMA REVISÃO DE LITERATURA
}

CERVICAL NECROTIZING FASCIITIS OF ODONTOGENIC ORIGIN. FROM ORIGIN TO TREATMENT: A LITERATURE REVIEW

\section{Tayná das Mecêrdes Gonçalves}

Graduada em Odontologia pela Faculdade de Odontologia da Universidade Federal Fluminense, Rio de Janeiro, RJ, Brasil e Pós-Graduanda do curso de especialização em Dentística da Faculdade de Odontologia da Universidade Federal Fluminense, Rio de Janeiro, RJ, Brasil.

\section{Maria Carolina Canadas Costa}

Graduada em Odontologia pelo curso de Odontologia da Universidade Iguaçu, Nova Iguaçu, RJ, Brasil. e Aluna do curso de Pós-graduação em Prótese Dentária da Universidade Iguaçu, Nova Iguaçu, RJ, Brasil.

\section{Suelen Cristina Sartoretto Lorenzi}

Professora das disciplinas de Cirurgia Bucal I, II e III da Universidade Iguaçu, Nova Iguaçu, RJ, Brasil. e Professora dos cursos de mestrado e doutorado em Odontologia da Universidade Veia de Almeida, Rio de Janeiro, RJ, Brasil.

\section{Marcelo José Uzeda}

Professor das disciplinas de Cirurgia Bucal I, II e III da Universidade Iguaçu, Nova Iguaçu, RJ, Brasil. e Professor das disciplinas de Cirurgia Oral Menor e Anestesiologia do curso de Odontologia da Universidade Federal Fluminense, Niterói, RJ, Brasil.

\section{Rodrigo Figueiredo de Brito Resende}

Professor das disciplinas de Cirurgia Bucal I, II e III da Universidade Iguaçu, Nova Iguaçu, RJ, Brasil. e Professor das disciplinas de Cirurgia Oral Menor e Anestesiologia do curso de Odontologia da Universidade Federal Fluminense, Niterói, RJ, Brasil.

Instituição na qual o trabalho foi realizado: Universidade Federal Fluminense, Niterói, RJ, Brasil.

Categoria: Revisão de Literatura

Tayná das Mecêrdes Gonçalves

Endereço: Rua Mario Santos Braga, 28 - Centro, Niterói - RJ, 24020-140

Telefone: (21) 96428-5736

E-mail: goncalvestay@gmail.com 


\section{RESUMO}

A Fasceíte Necrosante (FN) teve seu primeiro relato na época da guerra civil americana. Pode ser definida como uma infecção rara, com potencial de levar o paciente a óbito, onde acomete principalmente adultos e idosos, pacientes imunossuprimidos, portadores de diabetes miellitus e outros fatores sistêmicos, sem predileção por sexo. Estão presentes anaeróbicos e bactérias gram negativas nas feridas da FN. O sucesso no tratamento envolve o diagnóstico precoce, desbridamento cirúrgico radical de todo o tecido necrótico, antibioticoterapia parenteral de amplo espectro e medidas gerais de suporte agressivas. Os Cirurgiões Bucomaxilofaciais, muitas vezes, se deparam com casos de FN de cabeça e pescoço, pois as infecções odontogênicas são as causas mais comuns dessa infecção. Foi realizada uma revisão de literatura com o objetivo de fazer um levantamento sobre os tratamentos para Fasciíte Necrosante Cérvico-Facial por complicação de infecção odontogênica. O estudo tomou forma a partir de pesquisa bibliográfica apoiada em leituras selecionadas após a aplicação dos critérios de exclusão, que tenham ênfase em casos clínicos de Fasciíte Necrosante Cérvico-Facial por complicação de infecção odontogênica com resumo, tratamento proposto e evolução do paciente. A base de dados utilizada para busca dos artigos foram os Periódicos CAPES, Literature Analysis (PubMed/ MEDLINE) e Scientific Electronic Library Online (SciELO). Foram encontrados 44 artigos e, considerando os critérios de inclusão e exclusão e as variantes necessárias, foram incluídos para análise apenas 21 artigos. Os fatores relacionados a condições sistêmicas prévias, idade e gênero sexual não são determinantes para o acometimento da doença. O tratamento padrão ouro consiste em antibioticoterapia, drenagem, desbridamento, irrigação, curativo e remoção do foco infeccioso. A antibioticoterapia, independentemente de sua forma de administração, é fator chave para o tratamento. Sendo importante associar as classes de antibiótico beta-lactâmicos e nitroimidazol para um maior índice de sucesso na evolução do paciente.

Palavras-Chave: Fasciíte Necrosante Cérvico-Facial; Odontologia; Infecção.

\section{ABSTRACT}

Necrotizing Fasciitis (NF) had the first report at the time of the American Civil War. It can be defined as a rare infection, with death potential, affecting principally adults and the elderly, immunosuppressed patients, diabetes mellitus patients and other systemic factors, without preference by gender. Anaerobics and Gram Negative bacteria are present in NF lesions. Successful treatment involves an early diagnosis, radical debridement of all necrotic tissue, broad-spectrum antibiotic therapy, and aggressive general supportive measures. Oral and Maxillo Facial Surgeons often encounter facial-cervical NF because Odontogenic infections are the most common causes of this infection. This study aims to carry out a literature review with the objective of surveying the treatments for cervical-necrotizing fasciitis due to the complication of odontogenic infection. The study was based on bibliographic research based on selected readings after applying the exclusion criteria, which emphasize clinical cases of Facial Necrotizing Fasciitis due to the complication of odontogenic infection with summary, proposed treatment and evolution of the disease. The database used to search the articles were the CAPES Journals, Literature Analysis and Scientific Electronic Library Online. We found 44 articles and, considering the inclusion and exclusion criteria, only 21 articles were included for analysis. Factors related to previous systemic conditions, age and gender are not determinant 
for disease involvement. The gold standard treatment consists of antibiotic therapy, drainage, debridement, irrigation, band Aid and removal of the infectious focus. Antibiotic therapy, apart of its forms of administration, is a key factor for treatment. It is important to combine the betalactam antibiotic and nitroimidazole classes for a higher success rate in patient outcome.

Key words: Necrotizing Cervico-Facial Fasciitis; Dentistry; Infection.

\section{INTRODUÇÃO}

A Fasceíte Necrosante (FN) foi relatada pela primeira vez na época na guerra civil americana. No entanto o termo FN tivesse foi mencionado pela primeira vez, em 1952, por Wilson, que denominou, assim, as infecções necrosantes em tecidos moles, em que a fáscia muscular é invariavelmente envolvida (FREIRE, et al.,2012; YADAV, VERMA e SACHDEVA 2012; CORTEZZI, 1995). Segundo a literatura, a Fasciíte Necrosante ainda pode definida como uma infecção rara, potencialmente fatal, acometendo principalmente adultos e idosos e sem predileção por sexo. Não existem dados confiáveis quanto a sua real incidência na população (JUNIOR, et al., 2011).

A princípio, a microbiologia da Fasceíte Necrosante Cérvico-Facial (FNCF) era atribuída à Streptococcus pyogenes e Staphylococcus aureus. Com o passar dos anos e consequente aperfeiçoamento das técnicas de cultura, anaeróbicos e bactérias gram negativas foram também constatadas na lesão (CORTEZI, 1995). De acordo com literatura, existem dois tipos de infecção necrosante dos tecidos moles (INST) que são geralmente reconhecidas. O tipo I é definido como uma infecção poli microbiana, enquanto o tipo II é uma infecção monomicrobiana ou poli microbiana associada ao estreptococo do grupo A (GAS), bactérias e germes encontrados normalmente na garganta e na pele de pessoas saudáveis (HELCHER e BAKLEY 2019; ABE et al., 2017; YADAV, VERMA e SACHDEVA 2012).

A incidência dessa infecção é conhecida por ser maior em pacientes imunossuprimidos, portadores de diabetes miellitus, oncológicos, portadores de insuficiência cardiovascular, transplantados e com outras alterações sistêmicas. Cirurgias menos invasivas, como extrações, podem levar indivíduos imunocomprometidos a FNCF. Além disso, os atrasos nas cirurgias com infecções prévias somadas a indivíduos de idade avançada podem levar a Fasciíte Necrosante Cérvico Facial. No caso de jovens saudáveis 
sem distúrbios pré-sistêmicos é incomum a sua manifestação (ABE et al.,2017; YADAV. 2012; RICALDE, ENGORFF e JANSISYANONT 2004).

Estudos mostram que a FN é uma infecção dos tecidos moles que geralmente se inicia na gordura subcutânea ou na fáscia superficial e rapidamente se espalha ao longo de planos fascias (PARK et al., 2012; YADAV, VERMA e SACHDEVA 2012; BUCAK et al.,2013).

Na região de face, essa infecção se alastra do sistema músculo-aponeurótico superficial, um plano fascial que é próximo aos músculos da expressão facial, e se prolonga a partir da face, do músculo frontal ao platisma (PARK et al., 2012).

A necrose nas regiões do platisma suprajacente, do tecido cutâneo e da pele ocorre devido a trombose e obstrução das arteríolas que passam através do platisma para irrigar o tecido sobrejacente. No início, essa infecção causa vesículas cutâneas e, posteriormente, uma descoloração roxa escura da pele sobrejacente devido a uma isquemia. E por fim, necrose aparente e divulsão da pele propõe debridamento cirúrgico de grandes áreas da ferida (HUPP, 2015).

O tratamento bem-sucedido envolve o diagnóstico precoce, desbridamento cirúrgico radical de todo o tecido necrótico, antibioticoterapia parenteral de amplo espectro e medidas gerais de suporte agressivas (JUNIOR et al., 2011; HUPP et al.,2015). Caso o paciente não seja tratado precocemente, fatalmente o mesmo pode desenvolver septicemia, insuficiência múltipla de órgãos e, consequentemente, ir a óbito (FREIRE et al., 2014).

Infecções odontogênicas são as causas mais comuns da Fasciíte Necrosante Cérvico Facial (FREIRE, et al.,2012). Logo, os Cirurgiões Bucomaxilofaciais são os profissionais com mais chances de se depararem com Fasciíte Necrosante de Cabeça e Pescoço. Sendo assim, é ideal que estejam aptos para o diagnóstico, atendimento otimizado e principalmente optar por um tratamento mais eficaz para combater essa grave infecção (CORTEZZI, 1995). 


\section{MATERIAL E MÉTODOS}

Foi realizada uma revisão de literatura objetivando um levantamento bibliográfico dos tratamentos realizados para o tratamento das Fasciíte Necrosante Cérvico-Facial por complicação de infecção odontogênica.

$\mathrm{Na}$ revisão bibliográfica, foram realizadas consultas nas bases de dados do Periódicos CAPES, Literature Analysis (PubMed/ MEDLINE) e Scientific Electronic Library Online (SciELO) com os seguintes critérios de inclusão: artigos dos últimos 20 anos (entre agosto de 1999 a agosto de 2019), nos idiomas português e inglês, que tenham ênfase em casos clínicos de Fasciíte Necrosante Cérvico-Facial por complicação de infecção odontogênica com resumo, tratamento proposto e evolução do paciente. Os critérios de exclusão utilizados no estudo foram: artigos que ultrapassem os 20 anos limite, que não sejam em português ou inglês, sem enfoque Buco Maxilo Facial e revisões de literatura. Os termos utilizados para a busca foram: Fasciíte Necrosante cérvico facial (facial cervical necrotizing fasciitis), Fasciíte Necrosante Cérvico-Facial de origem odontogênica (facial necrotizing fasciitis of odontogenic origin) e fasceíte necrotizante de origem odontogênica: relato de caso (necrotizing fasciitis of odontogenic origin: case report).

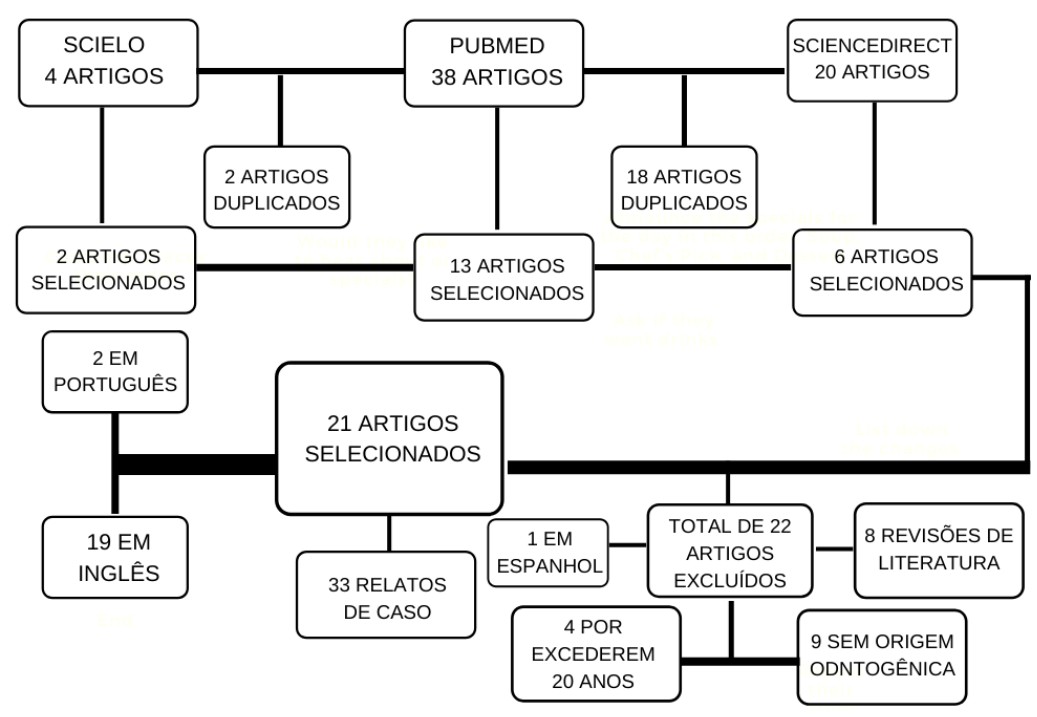

REVISTA FLUMINENSE DE ODONTOLOGIA - ANO XXVIII - N 56 - Julho / Dezembro 2021 


\section{Figura 1: Fluxograma dos artigos selecionados.}

\section{RESULTADOS}

Foram encontrados 44 artigos e, considerando os critérios de inclusão e exclusão e as variantes necessárias, foram incluídos para análise apenas 20 artigos, possuindo ao todo 34 relatos de caso. Na tabela 1 estão apresentados os artigos e respectivos autores localizados através dos descritores utilizados nas bases de dados e com período de publicação dos artigos, como estabelecido nos critérios de inclusão. A maior parte foi publicada em inglês (18 artigos), ainda que tenham sido estabelecidos como critério para a busca, artigos publicados em português e inglês.

Tabela 1: Dados clínicos, patologia associada, tratamento e evolução da Fasciíte Necrosante Cérvico-Facial.

\begin{tabular}{|c|c|c|c|c|c|}
\hline $\mathbf{N}^{\mathbf{o}}$ & Autor/Ano & $\begin{array}{l}\text { Idade/ } \\
\text { Sexo }\end{array}$ & Patologia Associada & Tratamento & Evolução \\
\hline 1 & $\begin{array}{l}\text { FREIRE et al } \\
2014\end{array}$ & $22 / \mathrm{F}$ & Não. & $\begin{array}{c}\text { Antibioticoterapia } \\
\text { venosa, drenagem do } \\
\text { abscesso, exodontia } \\
\text { múltiplas, } \\
\text { desbridamento } \\
\text { cirúrgico, irrigação e } \\
\text { curativo. }\end{array}$ & Alta \\
\hline 2 & $\begin{array}{c}\text { JUNIOR et al } \\
2011\end{array}$ & $37 / \mathrm{M}$ & Etilista crônico. & $\begin{array}{l}\text { Antibioticoterapia } \\
\text { empírica, exodontia } \\
\text { múltiplas e } \\
\text { desbridamento } \\
\text { cirúrgico. }\end{array}$ & Alta \\
\hline 3 & $\begin{array}{l}\text { LEYVA et al } \\
2013\end{array}$ & $51 / \mathrm{M}$ & $\begin{array}{c}\text { Diabetes Miellitus e } \\
\text { tabagista. }\end{array}$ & $\begin{array}{l}\text { Antibioticoterapia } \\
\text { venosa, drenagem do } \\
\text { abscesso, exodontia } \\
\text { múltiplas, antifúngico, } \\
\text { desbridamento e } \\
\text { irrigação. }\end{array}$ & Alta \\
\hline 4 & $\begin{array}{l}\text { ABE et al } \\
2017\end{array}$ & 32/M & Não. & $\begin{array}{l}\text { Antibioticoterapia } \\
\text { empírica parenteral, } \\
\text { desbridamento } \\
\text { cirúrgico, irrigação e } \\
\text { drenagem. }\end{array}$ & Alta \\
\hline 5 & $\begin{array}{c}\text { HELCHER, } \\
\text { BAKLEY } \\
2019 \\
\text { Caso } 1\end{array}$ & $18 / \mathrm{F}$ & Não. & $\begin{array}{l}\text { Antibioticoterapia } \\
\text { venosa de amplo } \\
\text { espectro, drenagem, } \\
\text { desbridamento e } \\
\text { irrigação. }\end{array}$ & Alta \\
\hline
\end{tabular}




\begin{tabular}{|c|c|c|c|c|c|}
\hline & $\begin{array}{c}\text { HELCHER e } \\
\text { BAKLEY } \\
2019 \\
\text { Caso } 2\end{array}$ & $15 / \mathrm{F}$ & Obesidade. & $\begin{array}{c}\text { Antibioticoterapia } \\
\text { venosa de amplo } \\
\text { espectro, drenagem e } \\
\text { irrigação. }\end{array}$ & Alta \\
\hline 6 & $\begin{array}{c}\text { PARK et al } \\
2012\end{array}$ & $36 / \mathrm{F}$ & Paralisia de Bell. & $\begin{array}{c}\text { Drenagem, } \\
\text { antibioticoterapia } \\
\text { venosa, desbridamento } \\
\text { cirúrgico e lavagem. }\end{array}$ & Alta \\
\hline 7 & $\begin{array}{l}\text { HOHLWEG- } \\
\text { MAJERT et al } \\
2005\end{array}$ & $75 / \mathrm{F}$ & $\begin{array}{c}\text { Diabetes de Miellitus } \\
\text { tipo II } \\
\text { descompensada, } \\
\text { hipertensa, poli } \\
\text { neuropatia, } \\
\text { hipercolesterolemia, } \\
\text { arteriosclerose } \\
\text { generalizada, } \\
\text { obesidade e etilista } \\
\text { crônico }\end{array}$ & $\begin{array}{l}\text { Exodontia, drenagem, } \\
\text { desbridamento } \\
\text { cirúrgico, irrigação e } \\
\text { antibioticoterapia } \\
\text { intravenosa. }\end{array}$ & Alta \\
\hline 8 & $\begin{array}{l}\text { THOMAS et al } \\
2012\end{array}$ & $31 / \mathrm{F}$ & Não. & $\begin{array}{l}\text { Antibioticoterapia } \\
\text { empírica, drenagem, } \\
\text { desbridamento } \\
\text { cirúrgico, } \\
\text { antibioticoterapia } \\
\text { venosa e oral. }\end{array}$ & Alta \\
\hline \multirow[t]{2}{*}{9} & $\begin{array}{c}\text { SAHOO e TOMAR } \\
2012 \\
\text { Caso } 1\end{array}$ & $56 / \mathrm{M}$ & $\begin{array}{l}\text { Etilista crônico e } \\
\text { Cirrose hepática. }\end{array}$ & $\begin{array}{c}\text { Drenagem, exodontias } \\
\text { múltiplas, } \\
\text { antibioticoterapia } \\
\text { empírica de amplo } \\
\text { espectro, } \\
\text { desbridamento } \\
\text { cirúrgico, irrigação, } \\
\text { curativo, } \\
\text { antibioticoterapia } \\
\text { venosa e oral. }\end{array}$ & Alta \\
\hline & $\begin{array}{c}\text { SAHOO e TOMAR } \\
2012 \\
\text { Caso } 2\end{array}$ & $36 / \mathrm{F}$ & Diabetes de Miellitus. & $\begin{array}{c}\text { Antibioticoterapia } \\
\text { empírica de amplo } \\
\text { espectro, } \\
\text { desbridamento } \\
\text { cirúrgico extenso e } \\
\text { antibioticoterapia oral. }\end{array}$ & Alta \\
\hline 10 & $\begin{array}{l}\text { KIM et al } \\
2016\end{array}$ & $61 / \mathrm{M}$ & $\begin{array}{l}\text { Artrite reumatoide } \\
\text { (tratado há } 7 \text { anos), } \\
\text { Osteoporose (tratado } \\
\text { com Alendronato há } \\
6 \text { meses), tabagista e } \\
\text { imunossuprimido. }\end{array}$ & $\begin{array}{c}\text { Drenagem, } \\
\text { antibioticoterapia } \\
\text { empírica, } \\
\text { antibioticoterapia } \\
\text { venosa e } \\
\text { desbridamento } \\
\text { cirúrgico. }\end{array}$ & Alta \\
\hline 11 & $\begin{array}{l}\text { MUHAMMAD et } \\
\text { al } \\
2014\end{array}$ & 26/M & Não & $\begin{array}{c}\text { Antibioticoterapia } \\
\text { endovenosa, } \\
\text { desbridamento } \\
\text { cirúrgico, mastectomia } \\
\text { da mama direita e } \\
\text { exodontia múltiplas. }\end{array}$ & Alta \\
\hline 12 & $\begin{array}{c}\text { YADAV, VERMA } \\
\text { e SACHDEVA } \\
2012\end{array}$ & $62 / \mathrm{M}$ & $\begin{array}{l}\text { Diabetes de miellitus } \\
\text { descompensado. }\end{array}$ & $\begin{array}{l}\text { Antibioticoterapia } \\
\text { empírica, múltiplas } \\
\text { exodontias, drenagem, } \\
\text { desbridamento }\end{array}$ & Alta \\
\hline
\end{tabular}




\begin{tabular}{|c|c|c|c|c|c|}
\hline & & & & $\begin{array}{l}\text { cirúrgico e antibiótico } \\
\text { tópico. }\end{array}$ & \\
\hline \multirow[t]{3}{*}{13} & $\begin{array}{c}\text { BUCAK et al } \\
2013 \\
\text { Caso } 1\end{array}$ & $36 / \mathrm{F}$ & Não. & $\begin{array}{l}\text { Desbridamento } \\
\text { agressivo e } \\
\text { antibioticoterapia } \\
\text { intravenosa. }\end{array}$ & Alta \\
\hline & $\begin{array}{c}\text { BUCAK et al } \\
2013 \\
\text { Caso } 2\end{array}$ & $81 / \mathrm{M}$ & Não. & $\begin{array}{l}\text { Desbridamento } \\
\text { agressivo e } \\
\text { antibioticoterapia } \\
\text { intravenosa. }\end{array}$ & Óbito \\
\hline & $\begin{array}{c}\text { BUCAK et al } \\
2013 \\
\text { Caso } 2\end{array}$ & $40 / \mathrm{F}$ & Não. & $\begin{array}{l}\text { Desbridamento } \\
\text { agressivo e } \\
\text { antibioticoterapia } \\
\text { intravenosa. }\end{array}$ & Alta \\
\hline 14 & $\begin{array}{l}\text { M. M., } \\
\text { CHIDZONGA } \\
2005\end{array}$ & $46 / \mathrm{M}$ & HIV positivo. & $\begin{array}{l}\text { Antibioticoterapia } \\
\text { intravenosa e } \\
\text { intramuscular, } \\
\text { drenagem, } \\
\text { desbridamento } \\
\text { cirúrgico, irrigação e } \\
\text { múltiplas exodontias. }\end{array}$ & Alta \\
\hline 15 & $\begin{array}{c}\text { FARRIER, } \\
\text { KITTUR e SUGAR } \\
2007\end{array}$ & $13 / \mathrm{M}$ & Não. & $\begin{array}{c}\text { Antibioticoterapia } \\
\text { intravenosa, drenagem, } \\
\text { exodontia e curativo. }\end{array}$ & Alta \\
\hline 16 & $\begin{array}{c}\text { RICALDE, } \\
\text { ENGORFF e } \\
\text { JANSISYANONT } \\
2004\end{array}$ & $14 / \mathrm{F}$ & Não. & $\begin{array}{c}\text { Drenagem cirúrgica, } \\
\text { antibioticoterapia de } \\
\text { amplo espectro, } \\
\text { desbridamento } \\
\text { cirúrgico, terapia } \\
\text { hiperbárica com } \\
\text { oxigênio, curativo a } \\
\text { vácuo e } \\
\text { antibioticoterapia oral. }\end{array}$ & Alta \\
\hline \multirow[t]{2}{*}{17} & $\begin{array}{l}\text { ULU et al } \\
2012 \\
\text { Caso } 1\end{array}$ & $50 / \mathrm{M}$ & Não. & $\begin{array}{l}\text { Desbridamento } \\
\text { agressivo extenso e } \\
\text { antibioticoterapia } \\
\text { intravenosa. }\end{array}$ & Óbito \\
\hline & $\begin{array}{l}\text { ULU et al } \\
2012 \\
\text { Caso } 2\end{array}$ & $51 / \mathrm{F}$ & Diabetes de Miellitus. & $\begin{array}{l}\text { Desbridamento } \\
\text { cirúrgico e } \\
\text { antibioticoterapia } \\
\text { intravenosa. }\end{array}$ & Alta \\
\hline \multirow[t]{4}{*}{18} & $\begin{array}{l}\text { UMEDA et al } \\
2002 \\
\text { Caso } 1\end{array}$ & $82 / \mathrm{F}$ & Sem dados. & $\begin{array}{l}\text { Desbridamento, } \\
\text { drenagem e } \\
\text { antibioticoterapia } \\
\text { intravenosa. }\end{array}$ & Alta \\
\hline & $\begin{array}{c}\text { UMEDA et al } \\
2002 \\
\text { Caso } 2\end{array}$ & $33 / \mathrm{F}$ & Sem dados. & $\begin{array}{c}\text { Desbridamento } \\
\text { extenso, drenagem e } \\
\text { antibioticoterapia } \\
\text { intravenosa. }\end{array}$ & Alta \\
\hline & $\begin{array}{c}\text { UMEDA et al } \\
2002 \\
\text { Caso } 3\end{array}$ & $28 / \mathrm{F}$ & Sem dados. & $\begin{array}{l}\text { Desbridamento } \\
\text { cirúrgico extenso, } \\
\text { drenagem e } \\
\text { antibioticoterapia } \\
\text { intravenosa. }\end{array}$ & Alta \\
\hline & $\begin{array}{l}\text { UMEDA et al } \\
2002\end{array}$ & $35 / \mathrm{M}$ & Sem dados. & $\begin{array}{l}\text { Desbridamento } \\
\text { cirúrgico extenso, }\end{array}$ & Alta \\
\hline
\end{tabular}

REVISTA FLUMINENSE DE ODONTOLOGIA - ANO XXVIII - N 56 - Julho / Dezembro 2021 


\begin{tabular}{|c|c|c|c|c|c|}
\hline & Caso 4 & & & $\begin{array}{c}\text { drenagem e } \\
\text { antibioticoterapia } \\
\text { intravenosa. }\end{array}$ & \\
\hline & $\begin{array}{l}\text { UMEDA et al } \\
2002 \\
\text { Caso } 5\end{array}$ & $67 / \mathrm{M}$ & Sem dados. & $\begin{array}{l}\text { Desbridamento } \\
\text { cirúrgico extenso, } \\
\text { drenagem e } \\
\text { antibioticoterapia } \\
\text { intravenosa. }\end{array}$ & Alta \\
\hline & $\begin{array}{l}\text { UMEDA et al } \\
2002 \\
\text { Caso } 6\end{array}$ & $46 / F$ & Sem dados. & $\begin{array}{l}\text { Desbridamento } \\
\text { cirúrgico extenso, } \\
\text { drenagem e } \\
\text { antibioticoterapia } \\
\text { intravenosa. }\end{array}$ & Alta \\
\hline & $\begin{array}{l}\text { UMEDA et al } \\
2002 \\
\text { Caso } 8\end{array}$ & $60 / \mathrm{F}$ & Sem dados. & $\begin{array}{l}\text { Desbridamento } \\
\text { cirúrgico extenso, } \\
\text { drenagem e } \\
\text { antibioticoterapia } \\
\text { intravenosa. }\end{array}$ & Alta \\
\hline & $\begin{array}{l}\text { UMEDA et al } \\
2002 \\
\text { Caso } 9\end{array}$ & $60 / \mathrm{M}$ & Sem dados. & $\begin{array}{l}\text { Desbridamento } \\
\text { cirúrgico extenso, } \\
\text { drenagem e } \\
\text { antibioticoterapia } \\
\text { intravenosa. }\end{array}$ & Alta \\
\hline 19 & $\begin{array}{l}\text { LORENZINI et tal } \\
2011\end{array}$ & $50 / \mathrm{F}$ & $\begin{array}{c}\text { Carcinoma ovariano e } \\
\text { Hepatite C. }\end{array}$ & $\begin{array}{l}\text { Antibioticoterapia } \\
\text { parenteral, drenagem } \\
\text { cirúrgica, múltiplas } \\
\text { exodontias, antifúngico } \\
\text { e medicação tópica. }\end{array}$ & Alta \\
\hline 20 & $\begin{array}{l}\text { JUNIOR et al } \\
2014\end{array}$ & $65 / \mathrm{M}$ & $\begin{array}{c}\text { Diabetes de miellitus } \\
\text { descompensado, } \\
\text { tabagista e etilista } \\
\text { crônico. }\end{array}$ & $\begin{array}{l}\text { Antibioticoterapia de } \\
\text { amplo espectro, } \\
\text { drenagem, múltiplas } \\
\text { exodontias, irrigação e } \\
\text { curativo. }\end{array}$ & Alta \\
\hline 21 & $\begin{array}{c}\text { ANTUNES et al } \\
2013\end{array}$ & $45 / \mathrm{F}$ & $\begin{array}{l}\text { Diabetes de miellitus } \\
\text { tipo I descompensado } \\
\text { e obesidade. }\end{array}$ & $\begin{array}{c}\text { Drenagem, } \\
\text { antibioticoterapia de } \\
\text { amplo espectro via } \\
\text { parenteral e } \\
\text { desbridamento } \\
\text { cirúrgico extenso. }\end{array}$ & Óbito \\
\hline
\end{tabular}

Legenda Tabela 1: F- feminino; $\mathrm{M}$ - masculino; $\mathrm{N}^{\circ}$, número de casos.

\section{REVISÃO DE LITERATURA}

No fator preponderante a idade de acometimento da doença (HELCHER e BAKLEY 2019), em seus dois casos, (FARRIER, KITTUR e SUGAR 2007; RICALDE, ENGORFF e JANSISYANONT 2004) tem ocorrências em pacientes jovens, antes dos 20/19 anos de idade. Os trabalhos de (FREIRE et al., 2014; JUNIOR et al., 2011; ABE et al., 2017; PARK et al., 2012; THOMAS et al., 2012; SAHOO e TOMAR 2012 caso 2, 
MUHAMMAD et al., 2014, BUCAK et al., 2013 caso 1; UMEDA et al., 2002 casos 2,3 e 4) apresentam casos com pacientes na faixa de 20/21 anos até os 40/39 anos.

Trabalhos como os de (JUNIOR et al., 2014; UMEDA et al., 2002 caso1; YADAV, VERMA e SACHDEVA 2012; BUCAK et al., 2013 caso 2; HOWLWEGMAJERT et al., 2005; KIM et al., 2016) apresentaram casos com pacientes idosos, todos na faixa acima dos 60 anos. Os outros trabalhos foram com relatos de pacientes na faixa etária de 40/41 até 59/60 anos.

No tocante quanto ao gênero de predileção para acometimentos da doença, os dados de (FREIRE et al., 2014; HELCHER e BAKLEY 2019; PARK et al., 2012; HOWLWEG-MAJERT et al., 2005; THOMAS et al., 2012; RICALDE, ENGORFF e JANSISYANONT 2004; LORENZINI et al., 2011; ANTUNES et al., 2013) tem exclusivamente o sexo feminino acometido com a FNCF. Os outros trabalhos têm o sexo masculino acometido com a patologia.

Em relação a patologia associada como origem para fascíite necrosante, os estudos de (FREIRE et al., 2014; ABE et al., 2017; THOMAS et al., 2012; MUHAMMAD et al., 2014; BUCAK et al.,2013; FARRIER, KITTUR e SUGAR 2007; RICALDE, ENGORFF e JANSISYANONT 2004) relataram não terem tido nenhuma condição sistêmica para o aparecimento da FN.

Os estudos de (LEYVA et al., 2013; HOHLWEG- MAJERT et al.,2005; YADAV, VERMA e SACHDEVA 2012; JUNIOR et al., 2014; ANTUNES et al., 2013) tem como origem agravante para a ocorrência de fascíite necrosante a diabetes miellitus com variações entre pacientes compensados ou não e com outros fatores sistêmicos associados ao quadro, como obesidade, tabagismo e etilismo.

Em relação aos outros trabalhos na literatura, foram observadas diferentes origens patológicas associadas, como cirrose hepática, estilismo crônico sem associação com outra doença, HIV, carcinoma ovariano, artrite reumatoide e paralisia facial de Bell. Outros artigos não tiveram dados sobre o quesito referente as condições sistêmicas associadas.

No que diz respeito ao tratamento, apesar de ter como base na literatura a antibioticoterapia, desbridamento cirúrgico, drenagem e remoção do foco infecioso, REVISTA FLUMINENSE DE ODONTOLOGIA - ANO XXVIII - No 56 - Julho / Dezembro 2021 
algumas variantes podem ocorrer, devido ao estado geral de saúde do paciente e/ou microbiologia da infecção. Em suas pesquisas (LEYVA et al., 2013; LORENZINI et al., 2011) utilizaram antifúngico no combate a infecção devido a presença de Candida albicans na ferida.

No tocante quanto a utilização de irrigação para tratar a ferida, (LEYVA et al., 2013; PARK et al., 2012; HELCHER e BAKLEY 2019 caso 1; FARRIER, KITTUR e SUGAR 2007) utilizaram somente o soro fisiológico 0,9\%. Nos estudos (HELCHER e BAKLEY 2019 caso 2; SAHOO e TOMAR 2012 caso 1), além de utilizarem a solução salina, água seguida de Clindamicina e solução de Darkin e peróxido de hidrogênio a 2\% e ácido acético glacial a 1\%, respectivamente. Já os autores (HOHLWEG-MAJERT et al., 2005; M. M., CHIDZONGA 2005) utilizaram Iodopovidona, para complementar o tratamento (HOHLWEG-MAJERT et al., 2005) utilizaram Betaisodona juntamente com metronidazol. (JUNIOR et al., 2014) em seus estudos utilizaram solução antisséptica para irrigar e tratar a ferida.

No levantamento dos trabalhos cuja a literatura fala dos curativos utilizados para o tratamento da ferida, é observado no estudo de (FREIRE et al., 2014) que empregaram pomada à base de sulfadiazina de prata $1 \%$ e nitrato de cério $0,4 \%$ e revestimento com Curatec® e bandagem. Já nos estudos de JUNIOR et al 2014 usaram curativos embebidos em uma preparação tópica de cloranfenicol. No seu estudo, (FARRIER et al., 2007) aplicaram Betadine em gaze encharcada e posteriormente Silastic espuma. (SAHOO e TOMAR 2012) fizeram somente a proteção da ferida com gaze. E, por fim, (RICALDE, ENGORFF e JANSISYANONT 2004) tem seus estudos lançaram mão do curativo à vácuo e terapia em câmara hiperbárica para tratamento da ferida.

Quando se fala antibioticoterapia utilizada no tratamento, os estudos levantados citam antibioticoterapia empírica, endovenosa, oral e tópica, dependendo da microbiota da ferida.

Trabalhos como os de (JUNIOR et al., 2011; KIM et al., 2016; YADAV, VERMA e SACHDEVA 2012; FREIRE et al., 2014; M. M., CHIDZONGA 2005) fizeram o uso de antibióticos das classes das beta-lactâmicos e nitroimidazol. Os autores (KIM et al., 2016; M. M., CHIDZONGA 2005) utilizaram também aminoglicosídeos como 
antibioticoterapia empírica. Os estudos de estudos de (ABE et al., 2017; THOMAS et al., 2012) lançaram mão da classe dos beta-lactâmicos, com (ABE et al., 2017) utilizando também as lincosaminas, assim como (RICALDE, ENGORFF e JANSISYANONT 2004) que complementou sua antibioticoterapia com aminoglicosídeos.

No tocante a antibioticoterapia intravenosa do tratamento FNCF para (BUCAK et al., 2013), nos casos 1, 2 e 3 foram utilizados somente beta-lactâmicos. Os autores (FREIRE et al., 2014; THOMAS et al., 2012; RICALDE, ENGORFF e JANSISYANONT 2004; PARK et al., 2011) lançaram mão de aminoglicosídeos e lincosaminas, com (THOMAS et al., 2012; PARK et al., 2011) complementaram a terapêutica com beta-lactâmicos em especial, (PARK et al., 2011) empregaram também na sua medicação nitromidazol.

Estudos de (JUNIOR et al., 2014; LORENZINI et al., 2010; ANTUNES et al., 2013) lançaram mão em sua terapêutica de antibióticos da classe dos beta-lactâmicos e nitromidazol. Ainda assim, complementaram o tratamento onde (JUNIOR et al., 2014) utilizaram aminoglicosídeos, (LORENZINI et al., 2010) glicopetídeos e (ANTUNES et al., 2011) lincosamidas na antibioticoterapia para o tratamento da FNCF.

No que se expressa sobre antibioticoterapia venosa, (ABE et al., 2017; HOHLWEG-MAJERT et al., 2005; RICALDE, ENGORFF e JANSISYANONT 2004) utilizaram beta-lactâmicos na terapêutica. Os autores (HOHLWEG-MAJERT et al., 2005) ainda fizeram uso de nitromidazol, igualmente a (SAHOO e TOMAR 2012), nos seus dois casos, onde complementaram com aminoglicosídeos. No trabalho de (RICALDE, ENGORFF e JANSISYANONT 2004) foram adicionadas lincosamidas. Ainda falando de aminoglicosídeos, (KIM et al., 2016) também utilizaram essa classe de antibióticos associadas com beta-lactâmicos. E finalmente, (MUHAMMAD et al., 2014) em sua pesquisa fizeram uso somente de lincosamidas na sua terapêutica.

Em relação antibioticoterapia usada no tratamento (M. M., CHIDZONGA 2005) utilizaram as classes das quilonas e das beta-lactâmicos. Os autores (FARRIER, KITTUR e SUGAR 2007) empregaram em sua terapêutica beta-lactâmicos e nitromidazol. Os estudos de (UMEDA et al., 2002) em seus 9 casos, apresentaram abordagens distintas. No caso 5 e 8 foram utilizados somente beta-lactâmicos. Nos relatos 6 e 9 empregou-se 
beta-lactâmicos e cefaloporinas. Nos episódios 1, 2, 3 e 4 foram aplicados betalactâmicos. Nos casos 1, 3 e 7 foram utilizados também cefaloporinas e lincosamidas. No relato 4 teve o complemento de lincosamidas e no 2, de azalídeos. Para o tratamento antibiótico (ULU et al., 2012), nos casos 1e 2 e fizeram uso de beta-lactâmicos e no caso 2 houve complementação com lincosamidas.

Nos casos de (JUNIOR et al., 2011; YADAV, VERMA e SACHDEVA 2012) a antibioticoterapia empírica utilizada foi resolutiva para o caso. $O$ que tange a antibioticoterapia oral (SAHOO e TOMAR 2012 caso 2); RICALDE, ENGORFF e JANSISYANONT 2004) utilizaram beta-lactâmicos. Os autores (RICALDE, ENGORFF e JANSISYANONT 2004) também fizeram uso de lincosaminas junto com os betalactâmicos. Em seu trabalho (THOMAS et al., 2012) lançaram mão das classes das cefaloporinas no tratamento antibiótico oral e (SAHOO e TOMAR 2012 caso 1) empregaram na terapêutica os azalídeos.

A remoção do foco infeccioso é algo de extrema importância no tratamento da FNCF. No caso dos trabalhos revisados (FREIRE et al., 2014; JUNIOR et al., 2011; LEYVA et al., 2013; HOHLWEG-MAJERT et al., 2005; SAHOO e TOMAR 2012 caso 1; MUHAMMAD et al., 2014; YADAV, VERMA e SACHDEVA 2012; M. M., CHIDZONGA 2005; FARRIER, KITTUR e SUGAR 2007; LORENZINI et al., 2011; JUNIOR et al., 2014) tiveram como objetivo exodontias de elementos dentários foco das infecções odontogênicas, após a estabilização do quadro clínico do paciente.

Ao abordar a questão do índice de óbitos por FN, os estudos de (BUCAK et al., 2013 caso2; ULU et al., 2012 caso 1; ANTUNES et al., 2013) relataram terem tido mortes por FN em seus casos. Os outros artigos revisados tiveram um índice de sucesso melhor, com seus pacientes tendo alta após o tratamento.

\section{DISCUSSÃO}

Segundo (ABE et al., 2017; FREIRE et al., 2014; UMEDA et al., 2002; PARK et al., 2012; SAHOO e TOMAR 2012) a Fasciíte Necrosante Cérvico-Facial (FNCF) é caracterizada por necrose rápida, progressiva e generalizada da fáscia superficial, com 
necrose da pele subjacente. Normalmente, se inicia com infecção odontogênica e em alguns casos os pacientes podem apresentar dificuldade de deglutição e trismo.

Tal patologia para (FREIRE et al., 2014) possui uma predominância maior pela mandíbula em relação à maxila. Para os autores (SAHOO e TOMAR 2012; LORENZINI et al., 2011) os casos com origem odontogênica envolvem com mais frequência segundo e terceiro molares inferiores, mais comumente causada por infecção periapical. Isso ocorre devido as raízes desses dentes se estenderem próximas de espaços fásciais profundos. Através desses espaços a infecção pode atingir e envolver a região do mediastino, aumentando o risco de mortalidade.

Os estudos de (FARRIER, KITTUR e SUGAR 2007; YADAV, VERMA e SACHDEVA 2012) relatam que a FNCF pode afetar pacientes de todas as idades, sem predileção por sexo ou raça. Porém, para (FREIRE et al., 2014) FNCF varia de 12 a 82 anos, apresentando uma média de 45,2 anos. O sexo masculino, de uma forma geral, é mais afetado que o feminino, numa proporção de 3:1, respectivamente. Avaliando esses estudos concluiu-se que o sexo masculino pode é o mais acometido devido à resistência dos homens ao acompanhamento médico, muitas vezes os mesmos podem apresentar alterações sistêmicas de forma silenciosa o que favorece o desenvolvimento $\mathrm{e}$ complicação da FNCF. Já em relação a faixa etária, crianças são menos acometidas devido ao maior acompanhamento médico e menor necessidade de extrações dentárias quando comparada a faixa etária de meia idade. Adultos de uma forma geral, pelo tempo de vida e necessidades odontológicas estão sujeitos a exodontia principalmente dos elementos dentários envolvidos com maior frequência em infecções odontogênicas.

Nos ensaios de (FREIRE et al., 2014; HELCHER e BAKLEY 2019; BUCAK et al., 2013; SAHOO e TOMAR 2012; UMEDA et al., 2002) pacientes imunocomprometidos, com idade avançada, hipertensos, obesos, diabéticos, cardiopatas, HIV positivo, etilista crônico, estão mais predisponentes a desenvolverem FNCF. Esses fatores agravantes ocorrem pela relação do envolvimento sistêmico do paciente com determinada doença, o que já compromete suas defesas naturais principais responsáveis pelo combate dessa infecção, deixando o paciente susceptível a septicemia. Porém, os mesmos também indicam que apesar de doenças prévias facilitarem a evolução de tal patologia, a fascíite necrosante da cabeça e pescoço pode ocorre em qualquer indivíduo, 
mesmo que não possua uma doença subentendida. Isso pode ser explicado devido a condições ambientais alteradas, uso indevido e descontrolado antibióticos e condições sociais dos indivíduos.

No que diz respeito a irrigação para tratamento da ferida causada pela FN, autores como (LEYVA et al., 2013, PARK et al., 2012, HELCHER e BAKLEY 2019 caso 1; FARRIER, KITTUR e SUGAR 2007) fizeram uso somente de soro fisiológico 0,9\%. Nos casos de (HELCHER, BAKLEY caso 2; SAHOO e TOMAR 2012 caso 1) complementaram o tratamento com água seguida de Clindamicina e outros degermantes. Nos estudos dos autores (HOHLWEG-MAJERT et al., 2005; M. M., CHIDZONGA et al., 2005) a Iodopovidona esteve presente e para complementar o tratamento. Em estudo solo (HOHLWEG-MAJERT et al., 2005) utilizaram Betaisodona juntamente com metronidazol, diferentes do estudo realizado por (JUNIOR et al., 2014), onde utilizaram solução antisséptica para irrigar e tratar a ferida. Todos os relatos que obtiveram tratamento com irrigação, os pacientes receberam alta. Pode-se considerar que a irrigação é um dos passos importante na cicatrização e evolução do paciente e um diferencial para a finalização positiva do tratamento.

Um outro fator grande importância para o tratamento no da ferida é o curativo. $\mathrm{O}$ mesmo protege ferida causada pela FN e também ajuda no processo de cicatrização, otimizando o tempo de internação do paciente. Certos estudos que fizeram o uso de curativo, tiveram certas discrepâncias quanto ao tipo utilizado pelos autores. No trabalho de (JUNIOR et al., 2014) usaram curativos embebidos em uma preparação tópica de cloranfenicol. Já os autores (FREIRE et al., 2014) pomada à base de sulfadiazina de prata $1 \%$ e nitrato de cério $0,4 \%$ e revestimento com Curatec ${ }^{\circledR}$ e bandagem foi o padrão empregado. Outra variante do curativo foi com (FARRIER, KITTUR e SUGAR 2007) com Betadine em gaze encharcada e posteriormente o uso Silastic espuma na ferida, algo bem incomum já que o mesmo é constituído de poliuretano e funcionada na ferida aproximando suas bordas, algo que em infecções é mais comum ocorrer o processo de cicatrização por segunda intenção. Os estudos de (SAHOO e TOMAR 2012) foram mais simples quanto a proteção da ferida, utilizando somente gaze. E por fim, de uma forma mais moderna, (RICALDE, ENGORFF e JANSISYANONT 2004) lançaram mão do curativo à vácuo que causa vasodilatação arterial e, consequentemente, o aumento do 
fluxo sanguíneo nos tecidos, estimulando a formação de tecido cicatricial de granulação e terapia em câmara hiperbárica onde o aumento de oxigênio puro circulante no corpo promove uma série de efeitos terapêuticos no corpo como: normalizando a cicatrização de feridas crônicas e agudas; neutraliza substâncias tóxicas e toxinas, potencializa a ação de alguns antibióticos que viabilizam o tratamento da ferida que agem como adjuvantes na terapêutica na FN.

No que tange a antibioticoterapia empregada, a mesma foi separada de forma empírica venosa, venosa após exames de cultura e oral pós alta da internação. Autores como (ABE et al., 2017; THOMAS et al., 2012; YADAV, VERMA e SACHDEVA 2012; RICALDE, ENGORFF e JANSISYANONT 2004; M. M., CHIDZONGA 2005) utilizaram beta-lactâmicos na antibioticoterapia empírica. Tal classe de antibióticos foi escolhido por ser de amplo espectro e bactericida, apesar de ao longo do tempo, algumas bactérias adquiriram a capacidade de produzir enzimas, denominadas beta-lactamases, que são capazes de promover a hidrólise do anel beta-lactâmicos, inativando a ação destes antibióticos. Outros artigos utilizaram outras classes de antibióticos como complementação.

Através de uma análise dos estudos, foi obtido um levantamento dos antibióticos mais utilizados na antibioticoterapia contra a FNCF. Autores como (JUNIOR et al., 2011; KIM et al., 2016; YADAV, VERMA e SACHDEVA 2012; FREIRE et al., 2014; M. M., CHIDZONGA 2005) fizeram o uso de antibióticos das classes das beta-lactâmicos e nitroimidazol. Isso por que os beta-lactâmicos possuem amplo espectro de ação e o nitroimidazol por ação antibacteriana contra bacilos gram-negativos anaeróbicos (causam infecções oportunistas em pacientes imunocomprometidos), contra bacilos grampositivos esporulados (Staphylococcus aureus, corriqueiro na $\mathrm{FN}$ ) todos os cocos anaeróbios (Streptococcus pyogenes, também comum na FN). Devido à natureza poli microbiana da FN, a associação de dois ou mais antibióticos se faz necessária. Dessa forma, esses antibióticos não foram somente usados juntos, mas também associados a outros, porém empregados aquém quando comparada a associação citada.

No que diz respeito a antibioticoterapia oral após alta da internação, (THOMAS et al., 2012; SAHOO e TOMAR 2012 caso 2; RICALDE, ENGORFF e JANSISYANONT 2004) lançaram mão de bata-lactâmicos, porém (RICALDE, REVISTA FLUMINENSE DE ODONTOLOGIA - ANO XXVIII - N 56 - Julho / Dezembro 2021 
ENGORFF e JANSISYANONT 2004) complementaram o seu tratamento com lincosamidas, pois agem contra Streptococcus e Staphylococcus resistentes à penicilina e a grande número de anaeróbios Já (SAHOO e TOMAR 2012 caso 1) empregaram na terapêutica os azalídeos que infecções da pele e tecidos moles (músculos, tendões, gordura) e são combatem bactérias como Staphylococcus aureus presentes na FN. Tal conduta pode ser empregada como uma finalização do tratamento e completa eliminação das bactérias causadoras da infecção do organismo do paciente, a fim de evitar recidiva.

No que se expressa sobre a evolução dos pacientes nos casos levantados, obtiveram-se 3 óbitos. Nos relatos de (ULU et al., 2012caso 1; BUCAK et al., 2013 caso 2) foram descritos 1 óbito em cada relato e de pacientes do sexo masculino, o que vai de encontro ao achado no levantamento, onde grande parte dos acometidos eram mulheres, levando a aceitar que o número de óbitos seria melhor para esse gênero. E terceiro óbito foi relatado por (ANTUNES et al., 2013), de uma mulher. Tal fato pode ser enaltecido segundo (MELO et al.,2008) que reconhece o paradoxo entre a vulnerabilidade do homem, sua característica pouco descuidada quanto ao cuidado e a inexistência de abordagens apropriadas que levem em conta toda sua forma de ser no cenário social principalmente social, o que contribui para o maior número de óbito para esse gênero sexual.

No tocante a respeito sobre a faixa etária dos doentes, (ANTUNES et al., $2013 \mathrm{e}$ ULU et al., 2012 caso 1) citaram os indivíduos de meia idade como os acometidos pela FN. Já (BUCAK et al., 2013 caso 2) narraram um paciente idoso de idade avançada como acometido. Pode se pressupor que os pacientes de meia idade são os mais acometidos pelo fato de apresentarem uma grande variação no que se refere às condições sistêmicas, psicológicas e sociais, além de serem portadores de várias alterações decorrentes do processo natural de envelhecimento.

No que diz respeito a patologia prévia, os trabalhos de (ULU et al., 2012 caso 1); BUCAK et al., 2013 (caso 2) demonstraram que os indivíduos infectados não tinham uma patologia associada. Já (ANTUNES et al., 2013) mostraram que seu paciente apresentou doenças sistêmicas crônicas previamente a FN. Os autores (ULU et al., 2012 caso 1); BUCAK et al., 2013 caso 2) demostraram em seus estudos que apesar de patologias prévias viabilizarem a evolução e possível óbito devido a FN, não necessariamente eles REVISTA FLUMINENSE DE ODONTOLOGIA - ANO XXVIII - N 56 - Julho / Dezembro 2021 
ocorreram em sua maioria, demonstrando que pacientes saudáveis estão suscetíveis cada vez mais a FNCF, por distintos fatores, desde ambientais até fisiológicos.

Quando se associa os óbitos a ausência de irrigação e curativo para tratamento da ferida, os autores (ANTUNES et al., 2013; ULU et al., 2012 caso 1; BUCAK et al., 2013 caso 2) expuseram a carência dessas duas ferramentas no tratamento da FN. O que enfatiza a importância do mesmo no tratamento, auxiliando no processo de cicatrização e diminuição exposição da ferida a mais micro-organismos que inviabilizam na recuperação do paciente.

Em relação a antibioticoterapia empírica, os trabalhos de (ANTUNES et al., 2013; ULU et al., 2012(caso 1); BUCAK et al., 2013 caso 2) não fizeram o seu uso. A terapia empírica é um tratamento baseado em experiência prévias, os casos em que utilizaram antibioticoterapia empírica, obtiveram sucesso. Isso se dá pelo fato de já combaterem a infecção com antibióticos de amplo espectro enquanto exames de cultura eram finalizados a fim de conhecer a microbiota da FN em questão.

Na antibioticoterapia terapêutica, os autores (ANTUNES et al., 2013) lançaram mão da associação de beta-lactâmicos. Apesar de ser a melhor escolha de associação de antibióticos para tratamento FN, o paciente veio a óbito. Tal fato pode ser enaltecido, pois também o mesmo era portador de Diabetes de Miellitus tipo I descompensado e obesa, condições sistêmicas que afetam diretamente na resposta imunológica do paciente frente a infecção e consequentemente a sua evolução. O trabalho de (BUCAK et al., 2013 caso 2) utilizou somente beta-lactâmicos na sua terapêutica, insuficiente, apesar do seu amplo espetro, não é suficiente pela natureza poli microbiana da FN, necessitando quase sempre de uma associação com outro antibiótico. Já os autores (ULU et al., 2012 caso 1) fizeram uso de beta-lactâmicos e lincosamidas, apesar do amplo espectro dos beta-lactâmicos e da ação das lincosamidas, principalmente a Clindamicina no combate a bacteriostático com atividade contra Streptococcus e Staphylococcus, o paciente veio a óbito por sepse.

No que se articula sobre antibioticoterapia oral após alta do paciente, os trabalhos (ANTUNES et al., 2013; ULU et al., 2012 caso 1; BUCAK et al., 2013 caso 2), não puderam lançar mão de mais esse instrumento no tratamento, pois seus pacientes vieram a óbito. 
É notório segundo (PEREIRA et al., 2019) que conhecimento quanto ao tipo de bactéria prevalente nas infecções cérvico-faciais é atualmente de extrema importância para uma melhor abordagem a respeito da antibioticoterapia a ser empregada no tratamento, sempre fazendo uso de associações entre as classes.

\section{CONCLUSÃO}

Apesar de alguns autores afirmarem, fatores relacionados a condições sistêmicas prévias, idade e sexo não são determinantes para o acometimento da doença.

Drenagem, antibioticoterapia, desbridamento, irrigação, curativo e remoção do foco infeccioso, associados e aliados a irrigação e curativo essenciais, apresentaram resultados satisfatórios. Ainda assim há uma necessidade de novos estudos, a fim de consumar um protocolo de tratamento para tal enfermidade.

A antibioticoterapia, independentemente de sua forma de administração, é fator chave para o tratamento, pois tem a finalidade de combater a infecção e auxiliar o sistema imunológico do paciente. Sendo importante associar as classes de antibiótico betalactâmicos e nitroimidazol para um maior índice de sucesso na evolução do paciente.

\section{REFERÊNCIAS BIBLIOGRÁFICAS}

1. Freire, Fábio de Freitas Pereira, et al. "Fasceíte Necrotizante Facial Causada por Infecção Odontogênica." Revista de Cirurgia e Traumatologia Buco-maxilofacial 14.1 (2014): 43-48.

2. Júnior, Rui Medeiros, et al. "Cervical-thoracic facial necrotizing fasciitis of odontogenic origin." Braz J Otorhinolaryngol 77.6 (2011): 805.

3. Abe, Masanobu, et al "Cervical necrotizing fasciitis of odontogenic origin in a healthy young patient without pre-systemic disorders" Journal of Oral and Maxillofacial Surgery, Medicine and Pathology Volume 29, Issue 4, (2017) Pages 341-344. 
4. Hechler, B. L., and G. H. Blakey. "Necrotizing soft tissue infection following routine third molar extraction: report of two cases and review of the literature." International journal of oral and maxillofacial surgery (2019).

5. Park, Eugene, et al. "Ascending necrotizing fasciitis of the face following odontogenic infection." Journal of Craniofacial Surgery 23.3 (2012): e211-e214.

6. Thomas, Andrew J., et al. "Klebsiella pneumoniae cervical necrotizing fasciitis originating as an abscess." American journal of otolaryngology 33.6 (2012): 764766.

7. Hohlweg-Majert, Bettina, et al. "Cervicofacial necrotizing fasciitis." Diabetes research and clinical practice 72.2 (2006): 206-208.

8. Sahoo, N. K., and Kapil Tomar. "Necrotizing fasciitis of the cervico-facial region due to odontogenic infection." Journal of Oral and Maxillofacial Surgery, Medicine, and Pathology 26.1 (2014): 39-44

9. Kim, Dong Hwi, et al. "Ascending facial necrotizing fasciitis in a patient taking a bisphosphonate." Journal of Oral and Maxillofacial Surgery 75.2 (2017): 317321.

10. Muhammad, Joseph Kamal, et al. "The value of early intervention and a multidisciplinary approach in the management of necrotizing fasciitis of the neck and anterior mediastinum of odontogenic origin." Journal of Oral and Maxillofacial Surgery 73.5 (2015): 918-927.

11. Yadav, Sunil, Ajay Verma, and Akash Sachdeva. "Facial necrotizing fasciitis from an odontogenic infection." Oral surgery, oral medicine, oral pathology and oral radiology 113.2 (2012): e1-e4.

12. Bucak, Abdulkadir, et al. "Facial paralysis and mediastinitis due to odontogenic infection and poor prognosis." Journal of Craniofacial Surgery 24.6 (2013): 1953-1956.

13. Chidzonga, M. M. "Necrotizing fasciitis of the cervical region in an AIDS patient: report of a case." Journal of oral and maxillofacial surgery 63.6 (2005): 855-859.

14. Farrier, J. N., M. A. Kittur, and A. W. Sugar. "Necrotising fasciitis of the submandibular region; a complication of odontogenic origin." British dental journal 202.10 (2007): 607.

REVISTA FLUMINENSE DE ODONTOLOGIA - ANO XXVIII - N 56 - Julho / Dezembro 2021 
15. Ricalde, P., et al. "Paediatric necrotizing fasciitis complicating third molar extraction: report of a case." International journal of oral and maxillofacial surgery 33.4 (2004): 411-414.

16. Ulu, Sahin, et al. "Paralysis of cranial nerve and striking prognosis of cervical necrotizing fasciitis." Journal of Craniofacial Surgery 23.6 (2012): 1812-1814.

17. Umeda, Masahiro, et al. "Necrotizing fasciitis caused by dental infection: a retrospective analysis of 9 cases and a review of the literature." Oral Surgery, Oral Medicine, Oral Pathology, Oral Radiology, and Endodontology 95.3 (2003): 283-290.

18. Lorenzini, Guido, et al. "Cervical necrotizing fasciitis of odontogenic origin involving the temporal region-A case report." Journal of Cranio-Maxillofacial Surgery 39.8 (2011): 570-573.

19. Camino Junior, Rubens, et al. "Cervical necrotizing fasciitis of odontogenic origin in a diabetic patient complicated by substance abuse." Brazilian dental journal 25.1 (2014): 69-72.

20. Antunes, Antonio Azoubel, et al. "Extensive cervical necrotizing fasciitis of odontogenic origin." Journal of Craniofacial Surgery 24.6 (2013): e594-e597.

21. Rosa, Lâner, et al. "Odontogeriatria-a saúde bucal na terceira idade." Revista da Faculdade de Odontologia-UPF 13.2 (2008).

22. Hupp, James, Edward Ellis, and Myron R. Tucker. Cirurgia oral e maxilofacial contemporânea. Elsevier Brasil, 2015.

23. Cortezzi, Wladimir. "Infecçäo odontogênica oral e maxilofacial." Infecçäo odontogênica oral e maxilofacial. 1995.

24. Pereira, Isabelly, et al. "Atualização Sobre Etiologia E Terapia Das Infecções Cervicofaciais De Origem Odontogênica" Revista da Faculdade de Odontologia$U F F(2019)$

25. de Melo, Elza Machado, et al. "Eles morrem mais do que elas. Por quê?." Rev Méd Minas Gerais 18.4 Supl 4 (2008): S12-S18 